\title{
Consideraciones Metodológicas sobre Investigaciones Sensibles en Metodología Cualitativa
}

\author{
Alicia Pérez-Tarres ${ }^{1}$ \\ ${ }^{1}$ Universitat Autònoma de Barcelona, Espanha. \\ Karine David Andrade Santos ${ }^{3}$ \\ ${ }^{3}$ Universidade Federal de Sergipe, SE, Brasil.
}

\author{
Leonor María Cantera Espinosa ${ }^{2}$ \\ ${ }^{2}$ Universitat Autònoma de Barcelona, Espanha. \\ Joilson Pereira da Silva ${ }^{4}$ \\ ${ }^{4}$ Universidade Federal de Sergipe, SE, Brasil.
}

Resumen: Algunos/as autores/as estudian y reconocen la dificultad emocional, por parte de quienes participan en investigaciones científicas, de revivir y relatar historias cuando estas se producen en contextos de violencia sea en el ámbito que sea (escuela, pareja, trabajo, etc.). El presente trabajo expone las dificultades metodológicas surgidas en el marco de una investigación cualitativa sobre la violencia laboral en dos países, Brasil y España. El objetivo de este artículo es ofrecer a la comunidad científica y política un conjunto de estrategias metodológicas para sortear los obstáculos que aparecen en la realización de una investigación con temática sensible. Las conclusiones de este trabajo indican que es necesario revisar las prácticas metodológicas que se llevan a cabo durante el diseño e implementación del estudio, pues esto puede evitar un perjuicio a los/las participantes e investigadores/as.

Palabras clave: Técnicas de intervención, Entrevista en profundidad, Estrategias metodológicas, Ética de la investigación.

\section{Considerações Metodológicas sobre Investigação Sensível em Metodologia Qualitativa}

Resumo: Alguns autores estudam e reconhecem a dificuldade emocional, por parte daqueles que participam de pesquisas científicas, de reviver e contar histórias quando estas ocorrem em contextos de violência em qualquer ambiente (escola, casal, trabalho etc.). O presente trabalho expõe as dificuldades metodológicas que surgiram no âmbito de uma pesquisa qualitativa sobre violência no trabalho em dois países, Brasil e Espanha. O objetivo deste artigo é oferecer à comunidade científica e política um conjunto de estratégias metodológicas para superar os obstáculos que surgem na realização de pesquisas sobre temas sensíveis. As conclusões deste trabalho indicam que é necessário rever as práticas metodológicas que são realizadas durante o desenho e implementação do estudo, pois isso pode evitar danos aos participantes e pesquisadores.

Palavras-chave: Técnicas de intervenção, Entrevista em profundidade, Estratégias metodológicas, Ética na investigação. 


\title{
Methodological Considerations for Sensitive Research on Qualitative Methodology
}

\begin{abstract}
Some authors study and recognize the emotional difficulty, on the part of those who participate in scientific research, of reliving and telling stories when these occur in contexts of violence in any environment (school, couple, work, etc.). The present work exposes the methodological difficulties that arose in the framework of a qualitative research on workplace violence in two countries, Brazil and Spain. The aim of this article is to offer the scientific and political community a set of methodological strategies for overcoming the obstacles that appear in carrying out research on sensitive subjects. The conclusions of this work indicate that it is necessary to review the methodological practices that are carried out during the design and implementation of the study, as this may avoid harm to participants and researchers.
\end{abstract}

Keywords: Intervention techniques, In-depth interview, Methodological strategies, Research ethics.

\section{Introducción}

El presente texto propone la exposición de aspectos metodológicos relacionados con las peculiaridades que viven las personas que participan en investigaciones científicas, vinculados a temas considerados sensibles y propios de atención en políticas sociales y públicas. Tal como asevera Sandín (2003), en su capítulo sobre la investigación cualitativa en educación, este tipo de metodología traspasa muchas disciplinas, utilizando variedad de perspectivas y desarrollándose a través de multitud de métodos y recogida de datos diversos. Si nos centramos en aquellas investigaciones que abordan la problemática de la violencia contra las mujeres y el género, advertimos, además, cómo la existencia de "marcos previos interpretativos", entre los que debemos considerar las diferentes formas de abordar metodológicamente la problemática, influyen en la formulación de las políticas de igualdad en Europa (Bustelo, \& Lombardo, 2006). En este sentido, en un estudio de Arranz (2015) se hipotetiza que, por ejemplo, en el caso de España, el Estado controla, y de forma ostensible, el tratamiento científico del problema social de la violencia de género (p. 172).

Considerando así el alcance, trascendencia y peso que la investigación científica tiene dentro de la política social como ciencia normativa que tiene como objetivo el estudio de la realidad social para la acción política y por ende sobre la creación de políticas públicas (y, por tanto, sobre la vida de los y las ciudadanos/as), resulta sorprendente que al reali- zar una búsqueda exhaustiva sobre la temática de género y la violencia, encontremos un gran corpus de investigaciones que se ocupan de realizar diagnóstico y buscar soluciones al problema, pero en muy pocas ocasiones se resaltan o abordan aspectos relacionados con la forma de abordaje ética de estas temáticas.

Por otro lado, planteamos si es responsabilidad de las autoridades políticas y del Estado el generar leyes, informes de impacto, evaluaciones y/o protocolos para el abordaje de las problemáticas sociales, ¿Por qué se dificulta tanto, llegando a la casi total inexistencia de consideraciones metodológicas consensuadas sobre la forma de investigar y aproximarse a estas realidades?

Este artículo sintetiza diversas cuestiones que surgieron en el marco del trabajo de campo realizado en el contexto de la investigación doctoral Violencia, trabajo y salud, que fue llevado a cabo entre diciembre de 2016 y septiembre de 2017. La investigación (detallada a continuación) propició la emergencia de cuestiones relacionadas con la forma de diseñar el estudio, la elección de la metodología, la aplicación de las técnicas o instrumentos de análisis y su interpretación. A lo largo del presente texto se plantean, en primer lugar, cuestiones teóricas que nos van a permitir comprender en profundidad los fenómenos prácticos experimentados en esta investigación. En segundo lugar, cuestiones experienciales surgidas a lo largo del trabajo de campo; con ellas, podremos ver el alcance de lo que la teoría implica. 
Mediante este artículo nos proponemos compartir nuestras reflexiones y experiencia en torno a la práctica investigadora, y convertir la misma en consideraciones prácticas en torno a la investigación con temáticas sensibles. Asimismo, debemos señalar la necesidad de incluir los planteamientos que aquí se exponen en investigaciones futuras sobre violencia y género; en los protocolos de abordaje de la problemática de instituciones públicas y/o privadas; $y$, por último, en las políticas públicas de abordaje de este problema social.

\section{Diseño de la investigación}

La población participante fueron trabajadoras/ es involucrados en tareas de erradicación de la violencia de género en dos países: Brasil y España. Nos inclinamos por un estudio comparativo entre Brasil y España pues este nos permitía enriquecer la comprensión de los fenómenos estudiados, haciendo hincapié en aquello que, - culturalmente -, se ignora o se tiene en cuenta, la forma de abordaje, la perspectiva de los y las participantes y la multitud de variables relacionadas. Estos y estas trabajadores/ as se enfrentan a diversos retos profesionales, los cuales implican el trabajo desde distintos niveles de intervención contra la violencia machista; desde la prevención, se trabaja por visibilizar y concienciar a jóvenes y adultos en lo que supone una sociedad patriarcal, con un profundo arraigo de las desigualdades entre hombres y mujeres donde la violencia de género es la máxima expresión de la misma. Desde la intervención, se trabaja para revertir las consecuencias de la violencia machista en aquellas que la sufren, asumiendo la enorme responsabilidad de aminorar el dolor y tratar las consecuencias de esta lacra sobre la vida de las mujeres y sus hijos/as. Los y las profesionales implicados en el tratamiento de situaciones violentas -maltrato infantil, violencia machista, situaciones traumáticas, etc.- están expuestos/as a un alto grado de desgaste profesional, que puede ser causa de trastornos psicológicos y/o físicos (Arón, \& Llanos, 2004). No obstante, y añadido a lo que implica - emocionalmente - el trabajar con víctimas de violencia, los y las trabajadores de este sector se enfrentan con otros problemas que profundizan y acentúan sus dificultades: las características del precario mercado laboral (contratos temporales, jornadas parciales, horas extras no remuneradas, etc.) y la reproducción de dinámicas violentas dentro de los equipos (Cantera, 2002; Quiñones, Cantera, \& Ojeda, 2013).

La investigación Violencia, trabajo y salud nació como respuesta a la necesidad de continuar indagando en las variables que posibilitan la emergencia de violencia en tantos contextos como personas y grupos conocemos. El diseño de nuestro trabajo puso en la palestra una serie de situaciones que nos obligaron a tomar conciencia de un fenómeno: la sensibilidad de nuestros objetos de investigación. Nos plantemos las siguientes cuestiones: ¿Cuáles son los principales problemas que encontramos en nuestra práctica investigadora al trabajar con una temática sensible? ¿Cuál es la metodología más indicada para aproximarnos a la realidad de la persona cuando esta debe hablarnos sobre su intimidad? ¿Qué se debería tener en cuenta al investigar con esta temática?

\section{Elección de un marco teórico y metodológico: la investigación cualitativa}

Con el objetivo de comprender y visibilizar el escenario resultante de las situaciones mencionadas con anterioridad, esta investigación tuvo como pretensión conocer el mundo interior de los participantes: para ello, se eligió el marco de la investigación cualitativa. No obstante, este camino se emprendió a través de la reflexión en torno a cuestiones como ¿nos permiten las metodologías de las que disponemos y conocemos comprender lo que las personas quieren transmitirnos? ¿Somos capaces de, a través de estos marcos teóricos, representar estas realidades? ¿Qué ventajas y/o barreras nos brinda el marco de la investigación cualitativa?

En la actualidad, la mayoría de investigaciones son llevadas a cabo con la utilización de técnicas cualitativas y/o cuantitativas. Estas técnicas, integradas dentro de un método cualitativo o cuantitativo, se diferencian entre ellas fundamentalmente porque la cuantitativa estudia la asociación entre variables que pueden ser cuantificadas e interpretadas numéricamente, y la cualitativa estudia estas asociaciones en contextos situacionales (Pita, \& Pértegas, 2002). Las características de la investigación cualitativa, su posicionamiento frente a la lógica del conocimiento, la forma de diseñar e implementar los estudios y la forma de entender y abordar los fenómenos 
sociales, decantaron nuestra elección frente a otras opciones epistemológicas.

Respecto al instrumento utilizado, tras una búsqueda exhaustiva y la consideración de distintas técnicas - entrevista en profundidad, grupos de discusión, observación, análisis de documentos biográficos, etc. - dentro del marco de la investigación cualitativa, se abogó por la entrevista en profundidad. La entrevista se define como la conversación de dos o más personas - cara a cara - en un lugar determinado para tratar un asunto, formando parte de una manera de enfocar la realidad (a través del método inductivo) (López Estrada, \& Deslauriers, 2011).

Seleccionamos la entrevista teniendo en cuenta los objetivos que nos planteábamos al diseñar el estudio, las características de los participantes y la temática trabajada, así como lo anteriormente citado por López Estrada y Deslauriers (2011) sobre las ventajas de la entrevista en profundidad para acceder al ámbito privado de los participantes. Reflexionamos, además, sobre algunas experiencias previas en estudios con profesionales dedicados a la erradicación de la violencia, como la expuesta en el trabajo de Cantera (2002), sobre la relación asistencial como interacción social o las mencionadas por Quiñones et al. (2013) las cuales, nos advirtieron, de la importancia de utilizar un instrumento capaz de conservar la intimidad de la persona, así como de aproximarse a sus vivencias de una forma gradual y respetuosa. Según Quiceno (2008), preservar la seguridad de los y las participantes es muy importante cuando se trabaja con víctimas de violencia. La confianza y el clima de intimidad son elementos imprescindibles para una obtención de la información adecuada. Según Catela (2004), aun y con los esfuerzos de los y las investigadores/as por crear un escenario perfecto en el que el informante pueda dar su palabra en confianza, la posibilidad de que lo narrado sea compartido a través de la difusión académica altera y transforma las interacciones personales. El acceso a los relatos de las personas participantes es una ardua tarea para el investigador, más aún cuando los testimonios están relacionados con problemáticas nacidas de la violencia política, étnica, religiosa (Catela, 2004) o laboral. Consideramos la entrevista en profundidad como el instrumento adecuado, pues este contribuye al cuidado del participante permitiendo que la persona exprese lo que piensa, siente o prefiere, sin temor a ser juzgado o cuestionado (Varguillas, \& Ribot de Flores, 2007).

\section{El trabajo con temáticas sensibles}

El trabajo expuesto en estas líneas fue diseñado para ser llevado a cabo siguiendo la lógica de la metodología cualitativa, por tanto, se presupuso la flexibilidad del diseño frente a las tareas que sucedían conforme progresaba el estudio. En palabras de Alencar-Rodrigues, Cantera y Ojeda (2014, p. 91) "el trabajo cualitativo se hace durante el andar" lo cual supone avanzar en función de los obstáculos y la toma de decisiones.

Asimismo, algunos autores y autoras plantean que las investigaciones sociales sobre violencia como un conjunto de temáticas muy complejas para los científicos sociales (Castillejo, 2005). Esto implica la consideración de ciertos aspectos teóricos, metodológicos y éticos en el tratamiento de este tipo de trabajos.

No obstante, al inicio de nuestra investigación (en el diseño inicial del estudio o en la fase de acceso a los participantes), no se consideró la magnitud que el término "temática sensible" podría suponer; más aún, la investigación se puso en marcha sin que se hubiera verbalizado de una forma explícita el término "temática sensible". El trabajo de campo nos puso ante diversas situaciones que provocaron una toma de conciencia. Esta sensibilización se produjo a través de las siguientes circunstancias - las cuales serán desarrolladas en los siguientes apartados -; dificultades de acceso a los participantes a las/los participantes (entidades o asociaciones que al ver el guión de la entrevista deciden no participar, participantes que acceden y se retractan, etc.). Problemas durante la entrevista: por parte de los/ las participantes fueron silencios, evasivas o reticencia a hablar de ciertos temas (incomodidad al recordar, dolor de la vivencia, miedo a las represalias o gran necesidad de anonimato). Por parte del investigador, se produjo una dificultad en el acceso a la información requerida y cierto impacto emocional durante y tras la realización de la entrevista. Por último, el reflejo de estas situaciones sobre los resultados finales del trabajo.

\section{¿Qué es un trabajo con temática sensible?}

Lee (1993) en su extenso trabajo titulado "doing research in sensitive topics" expone tres situaciones bajo las cuales una temática concreta puede ser considerada como un tema sensible. La primera se refiere 
al sentimiento de amenaza que la investigación pueda generar en el o la participante; se relaciona con áreas privadas o estresantes de la vida personal. La segunda tiene relación con la posibilidad de que la información extraída en el estudio pueda ser revelada o pueda incriminar de alguna forma a la persona. Está relacionada con el control social. Por último, la tercera implica la consideración de los intereses de personas o instituciones poderosas que puedan ejercer o ejerzan la coerción o dominación. Asimismo, se puede considerar sensible también aquella investigación cuyos resultados puedan suponer unas consecuencias o implicaciones tanto para los participantes como para los individuos a los cuales representan los hallazgos (Sieber ,\& Stanley, 1988).

En un trabajo de Díaz Fernándes, Ruiz Rodríguez, González Agudelo y Gallardo García (2012), se define aquello que se puede considerar como "temática sensible" afirmando que influye la delicadeza del tipo de información o las preguntas que se han de formular pues puede molestar a quien se entrevista. Asimismo, aducen al carácter sensible de cualquier investigación en ciencias sociales, pues siempre se extrae información que antes no era visible y se revela parte de la intimidad de una persona (lo cual puede generar suspicacia o desconfianza). El investigar con una temática sensible supone, entre otras cuestiones, identificar los aspectos que las personas participantes desean mantener en secreto. También, considerar los intereses de algunas organizaciones o el mismo Estado en mantener oculta cierta temática (Ramírez-Pereira, 2017). Sobre esto último, es interesante considerar para la investigación aquellos aspectos que desean mantenerse en secreto - y a qué intereses responde este hecho - en el momento de imaginar, plantear y diseñar la investigación.

Es por ello (entre otros factores) que algunas investigaciones (Castillejo, 2005; Catela, 2000; Quiceno, 2008; Romero, 2008) en el ámbito de la violencia se centran en desenmarañar aquello indecible, opaco y en lo que implica la dificultad de acceso a estos relatos.

\section{Persona entrevistada y quien entrevista}

Alencar-Rodrigues et al. (2014) afirman en su trabajo que la especificidad de la temática de violencia requiere que el equipo de investigación atienda al efecto que puede tener la entrevista en la vida de los y las participantes e investigadores(as). Por tanto, es importante tener en cuenta aquello que la participación implica para los y las entrevistados/as; exponerse, desnudar sus sentimientos, dejar ver su parte más íntima, compartir sus experiencias. Estos actos llevan implícita una dificultad añadida a la participación (inversión de tiempo, esfuerzos para comprender el trabajo, etc.), pues la misma expresión emocional implica la movilización de procesos psicológicos que no siempre se está dispuesto - o preparado - a afrontar.

La realización de las entrevistas de esta investigación se llevó a cabo en el ambiente que la persona consideró más oportuno para su comodidad e intimidad. En todo momento, las investigadoras respetaron estos elementos para maximizar la participación en el estudio y tratar cuidadosamente a las/los participantes. Existe cierto riesgo de sufrir un impacto psicológico por participar y exponer tu historia en una investigación sensible (MacCosker, Bernard, \& Gerber, 2001) por lo que las reticencias de esta participación se hacen patentes en diversos momentos del transcurso del estudio (sobre todo en la fase de acceso a los participantes, conformación de la muestra y entrevista en profundidad). El primer acercamiento con estas personas se realizó con una llamada telefónica (los contactos se establecieron mediante un muestreo en bola de nieve), por lo tanto, entrevistadora-entrevistado/a no se conocían previamente ni tenían información personal del/la otro/a (más allá de la administrada durante la llamada referida al carácter de la investigación y credenciales de la entrevistadora).

Así pues, se hicieron arduos esfuerzos por crear un clima de confianza en el que la persona pudiera expresar sus vivencias frente a una persona desconocida (en este caso, quien realizaba la entrevista). Durante las entrevistas se observaron diversos fenómenos relacionados con la movilización o recuerdo de las experiencias traumáticas de los/las entrevistados que acabaron redundando o teniendo un efecto sobre ellos/as mismos/as, las entrevistadoras y la investigación. Estas situaciones se repitieron no solo en la mayoría de entrevistas realizadas, sino también en los dos contextos culturales que estábamos trabajando: Brasil y España. En Brasil, algunas/os entrevistadas/os afirmaron que no era fácil hablar sobre sus experiencias de acoso o violencia en el trabajo. Esta dificultad se expresó a través de diversas emociones como el miedo o temor a contar algún episodio en concreto, el llanto al hablar sobre 
algunas experiencias o el rencor y la rabia no trabajada que se apreciaba en algunos discursos al hablar de los/las acosadoras.

En este fragmento extraído de una entrevista realizada a una trabajadora en Brasil se observa como la persona moviliza sus recuerdos y emociones al relatarlo para la investigación:

Entrevistada: Pero, yo no perdoné, de hecho. Yo no he perdonado al día de hoy. Me encontré con ella hace un tiempo, ahí, en el centro. Hice que no la vi. No perdoné porque soy así, me resulta difícil perdonar. Esto es muy malo; no perdonar. ¿Por qué sigues vivenciando aquello, yo ahora hablando contigo, yo lo estoy viviendo, lo estoy recordando todo y me enfado, lo entiendes? Es horrible, espero que lo pueda superar.

Los y las participantes de España también expresaron estos sentimientos, a lo que se añadió evitar responder a algunas de las preguntas planteadas. Algunas de las personas entrevistadas en ambos contextos relataron experiencias de violencia física y psicológica. Nuestra entrevista estuvo articulada en dos ejes principales: por un lado, la salud, apoyo social y autocuidado y, por otro lado, las experiencias de violencia en el trabajo. De manera general, las/los entrevistados quedaron desahogados y tranquilos al hablar sobre el primer bloque, pero al tratar los temas del segundo bloque (vivencias de violencia) se observó mucha tensión y temor. Asimismo, no solo las emociones captadas (y recogidas mediante diario de campo) por las entrevistadoras hicieron patentes estas dificultades al hablar de ciertos temas, sino también las evasivas y los silencios que se repitieron ante ciertas preguntas. Díaz Fernádez et al. (2012) afirman que hay cierta información que quiere ser ocultada por las personas y comprender estas necesidades y sus miedos puede ayudar al investigador a entrar en el objeto de estudio.

Conforme fuimos comprendiendo lo anteriormente citado, se desarrollaron estrategias para salvaguardar "el equilibrio psicológico" de quien hablaba y maximizar la validez de la información extraída. Además, esto nos ayudó a comprender la importancia, el peso, el desgarro que esas vivencias tenían para la persona que entrevistábamos y, por lo tanto, la trascendencia que tenían para la investigación. En un trabajo de Quiceno en 2008 con víctimas de la guerra, se afirmó que, pese a los esfuerzos en las estrategias del diseño metodológico para afrontar las dificultades de la investigación, la vulnerabilidad de esas víctimas solo conseguía aminorarse. El dolor y agravio dejado por la guerra puede sobrepasar cualquier estrategia $o$ voluntad por no hacer más dolorosos los recuerdos. En otro trabajo, realizado por la OMS (1999) se apunta a las dificultades de entrevistar en ciertos asuntos; por ejemplo, se afirma que existe la creencia de que la violencia contra las mujeres es un tema demasiado delicado para ser objeto de estudio ya que la vergüenza, la culpa o el temor pueden evitar que esas mujeres hablen sobre sus experiencias.

Observamos, también, las repercusiones de esta situación para aquel que pregunta, el o la entrevistador/a, ya que los silencios, las narraciones del otro y los vacíos de información penetran en la conciencia ética de quien los escucha (Romero, 2008). En un estudio sobre víctimas de violencia de Quiceno (2008), se destacó la complejidad, por parte de quien investiga, de manejar y afrontar las propias emociones. En esta misma investigación, se consideró la complejidad de escuchar, sentir, comprender y dimensionar el dolor del otro y se afirmó que esto eran efectos que tenía el trabajo de campo sobre los investigadores.

Respecto a nuestra experiencia durante la investigación, tratar y hablar sobre los conflictos y el acoso en el trabajo supuso una experiencia desafiante; nos remitió a algunas experiencias en nuestros propios contextos de trabajo. Entre los efectos que la realización de este trabajo puede ocasionar en los y las investigadores/as encontramos el citado por Ramírez-Pereira $(2017$, p. 8) quien afirma que "el trabajar con temas sensibles puede resultar sanador para el investigador ya que es posible que lea su propia historia en la historia del otro". La realización de las entrevistas exigió un esfuerzo por manejar y conducir las emociones que surgieron en los momentos iniciales del trabajo de campo. Conforme avanzó la investigación y aumentaron los recursos internos (asimilación de las historias, conexión con uno mismo o motivación por la temática) este esfuerzo se recondujo hasta conseguir un desempeño emocional adecuado. Comprendimos y experimentamos lo que Quiceno (2008) afirma cuando dice que una mirada "desde fuera" no existe en los contextos en los que la investigación implica una alta carga emocional, y que es preciso interrogarse de forma consciente para encontrar un equilibro entre la proximidad y la lejanía que el trabajo de campo requiere. 
Así pues, asumimos que debíamos aceptar la influencia del investigador sobre el planteamiento de la investigación (por la subjetividad que lo cualitativo implica) y, de modo inverso, considerar el impacto del relato sobre quien investiga. Estas sinergias acentúan la necesidad de trabajar desde unos marcos éticos previos al inicio del trabajo de campo que permitan desarrollar la investigación de una forma ética, eficaz y respetuosa. La ética de la colaboración, citada por Castillejo (2005), requiere no solo de un compromiso profundo, sino también de la autorreflexión de quienes investigan, para evitar la reproducción de dinámicas violentas de aquellos que nos antecedieron.

\section{¿Cómo influye la sensibilidad del tema al transcurso de la investigación?}

No es posible hablar de relaciones predeterminadas (como la de entrevistado-entrevistador) sin que lo "impreciso, lo dudoso, lo confuso, lo oscuro, lo borroso y lo imposible se haga presente" (Castellano, 1998, p. 2).

Encontramos, en la literatura actual, diversos trabajos con víctimas de violencia en ámbitos como la guerra, el holocausto o los desplazamientos forzados que dan luz, trabajan y tratan de profundizar en algunas cuestiones que emergen en las investigaciones en las que se trata con muestras de sobrevivientes, sus familiares y/o con víctimas de violencia de cualquier tipo. Por ejemplo, en 2005, Castillejo, en su trabajo sobre el Apartheid en Sudáfrica trató de entrevistar a unas mujeres cuyos hijos habían sido asesinados en el transcurso de ese gravísimo problema social. En esta investigación, se afirmó que el impacto de estos sucesos fue tan lesivo para esas madres que la simple idea de relatarlo les hacía temer un -nuevo - ataque de pánico. En este caso, surgió la necesidad de dejar los recuerdos dolorosos en el ámbito de lo puramente íntimo. Quiceno, en 2008, afirmó que para el trabajo de campo es necesario reconocer la forma y el escenario en que las víctimas relatan sus testimonios, y de qué manera aquello que prefiere callarse o ser silenciado da razones a las preguntas de investigación. Estos silencios se pueden expresar como límites al acceso a la información por parte de los investigadores sociales (Romero, 2008). Asimismo, en un estudio de Díaz Facio-Lince, Molina Jaramillo y Marín (2014) se asevera que no solo se debe atender a los recuerdos y a los relatos de los/ las participantes, sino también a sus silencios y a las referencias del olvido, pues son muy importantes para la comprensión de su vivencia. En la línea de lo que se concluye en las investigaciones mencionadas con anterioridad, pudimos comprobar en nuestro propio trabajo cómo emergían estos fenómenos. Mientras se efectuaba la entrevista, observamos cómo algunas/os participantes trataban de evitar responder a preguntas relacionadas, sobre todo, con las vivencias violentas en sus contextos de trabajo. En otras ocasiones, hubo largo silencio tras la realización de la pregunta o el participante dudó o respondió de forma contradictoria a lo anteriormente explicado. En las transcripciones de las entrevistas quedaron plasmadas algunas de estas dificultades relacionadas con los temores de los participantes a contar ciertos detalles de su historia. El paso del relato íntimo al compartido y la difusión académica pueden afectar la confianza y naturalidad de quien es investigado. En palabras de Catela (2004, p. 71):

lo no "dicho", lo "censurado", lo "corregido" están íntimamente ligados a la significación que toma el hecho del paso de la palabra privada al mundo de lo público, donde los condicionamientos sociales, culturales y políticos atraviesan la expresión de las historias singulares y de sus identidades.

El siguiente ejemplo, extraído de la entrevista a uno de los participantes en Barcelona-España, es ilustrativo:

Entrevistado: Sí, exacto... a veces digo que este tipo de entidades (que luchan contra la violencia) es como el hijo, el cruce entre Teresa de Calcuta y el Ronald McDonald. Empieza con una cosa que tiene que ver con el servicio a los demás y se convierte en una empresa que quiere más, más poder y más... más gente, más... y esta ambición con lo otro es a veces difícil de encajar. Ahí se generan choques un poco esquizofrénicos en el sentido en que están tirando para dos sitios diferentes... y en esta, en esta última entidad he vivido situaciones de violencia, a ver cómo te diría... ah no sé, mmm....uf... no sé.... me cuesta recordarlo... si recuerdo haberlo pasado mal... pero ni idea... no sé.

Observamos que la persona entrevistada empieza a explicar diferentes situaciones que ha vivido en la 
entidad en la que trabaja en la actualidad a continuación duda y acaba por reconocer que no recuerda con exactitud, que no sabe qué sucedió. ¿Qué se puede inferir en este fragmento? ¿Cómo podemos interpretar este olvido?

En otro ejemplo extraído de nuestras entrevistas, la participante evita hablar directamente sobre los conflictos que ha vivido en primera persona en su puesto de trabajo:

Entrevistadora: ¿Qué formas de violencia te has encontrado tú en tu ambiente laboral?

Entrevistada: Para mí hay un tipo de personas... hay un tema en relación a las personas que tienen un carácter más perverso y que a veces están en estos centros y en estos servicios... y... ostia es súper difícil, ahora estoy pensando en, no sé, por ejemplo un servicio, en el que yo he coincidido poco, pero siempre las trabajadoras estaban fatal por tener esa directora y que está allí y que es difícil tenerla ahí y es un desprecio hacia el equipo, una falta de respeto... una relación realmente difícil y realmente violenta.

Asimismo, cuando se le pregunta por las repercusiones de los conflictos laborales, relata lo siguiente:

Entrevistadora: Entonces, ¿hay algunas repercusiones y consecuencias que haya tenido este trabajo para tu salud o en relación a los conflictos?

Entrevistada: Yo, para mí, hombre, los conflictos generan una angustia impresionante y un malestar impresionante, un no dormir y un absolutamente de todo pero esa es la violencia vivida como víctima... ¿no? No la atención (con las mujeres). Dijéramos la violencia en el equipo es terrible, es devastadora... en mi caso ha sido mucha angustia, por primera vez en mi vida no comer y... y dormir con mucha dificultad.

En este último ejemplo, pese a los esfuerzos de la investigadora (cuidar la comunicación verbal y no verbal, remarcar aspectos sobre la confidencialidad del trabajo, dar tiempo para responder, crear un clima de intimidad, contener la emoción, entre otros) no fue posible que la participante relatara aquello que había vivido como víctima. Únicamente pudimos acceder a las consecuencias que esa violencia había tenido para ella.
$\mathrm{Al}$ conocer la dificultad que experimentaban las personas participantes frente a ciertos temas, nos planteamos las causas de tanta reticencia. Tras el análisis de la información, observamos que la vivencia de experiencias traumáticas, violencia o acoso laboral en el lugar de trabajo, podía tener profundas repercusiones para la persona y el testimonio o el revivir de estas experiencias podía resultar difícil y doloroso para quien lo había sufrido. Autores como Goodrum y Keys (2007) o Declan (2014) afirmaron que la revisión de las experiencias (de acoso) a lo largo de una entrevista podía resultar traumática para el/la investigador/a y el/la participante. Los resultados de estos trabajos coinciden con los nuestros.

Por otro lado, el temor a señalar a personas, asociaciones o entidades concretas o, simplemente, poner en tela de juicio la labor que se realiza en estos centros, fueron obstáculos para los/las participantes al hablar de sus vivencias. Observamos un impacto en el plano emocional, pero también en el racional (o intelectual), al tener dificultades para integrar las experiencias violentas en este contexto laboral cuando se producen en un ambiente en el que se trabaja para erradicar la violencia machista.

\section{Consideraciones prácticas}

Conforme avanzó el trabajo de campo de esta investigación y experimentamos la dificultad de acceso al relato de algunos/as participantes, las emociones que surgían frente a la entrevista y el impacto que podía tener esto para los resultados de la investigación, se examinó la literatura actual referente a las técnicas metodológicas para poder dar una respuesta adecuada ante estas situaciones. En adelante, se propone un decálogo de consideraciones metodológicas extraídas de diversos trabajos y algunas técnicas propias puestas en marcha en nuestra investigación teniendo en cuenta lo que McCosker et al. (2001) indican, al aseverar que una estrategia genérica es inadecuada en su aplicación a todas las situaciones de una investigación "sensible" dado que se debe ser especifico en cada circunstancia o caso concreto:

1. Tomar medidas para asegurar la seguridad física, moral y legal de los participantes durante y una vez finalizada la fase de trabajo de campo. Esto supone garantizar la confidencialidad de los datos extraídos así como el anonimato de sus identidades (Díaz Fernández et al., 2012). Nues- 
tro trabajo añade a esta recomendación el mostrar una actitud respetuosa, empática sin emitir juicios de valor o utilizar lenguaje que pueda resultar estigmatizador.

2. Aclarar los objetivos, fases y proceso de la investigación al/la entrevistado(a). Es menester dejar claro a los participantes que la relación finaliza al término de la investigación (Ramírez-Pereira, 2017). Por lo tanto, es importante clarificar los límites de lo que supone la relación entre participante-investigador/a.

3 . Tener en cuenta estrategias que permitan preservar la estabilidad psicológica del investigador/a (no dañarlo) al mismo tiempo que se salvaguarda la fiabilidad de los datos (Díaz Fernández et al., 2012). En 1995, McCosker, propuso algunas estrategias para ayudar al investigador y/o investigadora en su práctica profesional en el marco de una investigación sobre mujeres y abuso: no hacer más de una investigación por semana, procurar que la entrevista sea en horario matutino, proponer a otra persona para realizar la transcripción de las cintas, no escuchar sin descanso más de una hora de entrevistas o no leer literatura sobre la temática mientras se recopila información en el campo. A esto, nuestra propuesta añade el autocuidado activo, que supone procurar y hacer consciente la necesidad de un correcto cuidado por parte de las/los investigadores(as) antes y una vez acaba la entrevista; actividades como el ejercicio físico, la relajación, la realización de actividades gratificantes o las supervisiones, entre otros, han mostrado su eficacia en trabajadores que abordan temáticas de violencia.

4. Tener conciencia de las consecuencias relacionadas con preguntar sobre conocimientos, actitudes o experiencias de vida relacionadas con fenómenos "sensibles" que puede tener la investigación para las personas entrevistadas (McCosker et al., 2001).

5. Para atender a las respuestas emocionales de las personas participantes, entre las estrategias más citadas encontramos el dar tiempo a la persona entrevistada para llorar o expresar emociones significativas, así como reconocer la importancia de esto para su bienestar (McCosker et al., 2001). Hay que tener en cuenta, sin embargo, lo que Schraiber, Oliveira y Couto (2009) afirman cuan- do nos dicen que es importante mantener la postura de investigación, para evitar que la situación de la entrevista pueda convertirse en una terapia para la persona entrevistada.

6. Comentar las fortalezas y aspectos positivos del o la entrevistado/a, así como dar la oportunidad y saber leer las señales que indican que la persona está demasiado angustiada como para continuar con la entrevista. La falta de respuesta ante estos aspectos puede llevar a una pérdida de confianza por parte del/a entrevistado/a respecto a quien está investigando (McCosker et al., 2001).

7. Es importante tener en cuenta el lugar donde se realiza la entrevista. Es mejor que esta se realice en un ambiente privado. Además, la participante debe tener el derecho a cambiar el día y lugar de la entrevista, hasta encontrar el que considere más fiable y seguro (OMS, 1999).

8. Conocimiento profundo de las temáticas que van a tratarse durante la entrevista y entrenamiento de la/el entrevistadora/or en la técnica para poder cambiar de tema o finalizar la entrevista si fuera oportuno.

9. Tratar de clarificar aquellos temas en los que se ha podido generar confusión o evitación, acompañando el recuerdo de la persona sin ejercer presión por parte de quien pregunta (Quiceno, 2008).

10. Según Cantera (2018), es responsabilidad de quien interviene en la faceta que sea: investigación, atención directa, y/o intervención del tipo que sea; el tener un listado de referencias de lecturas que puedan ayudar a la persona con la que se está interactuando a entender lo que está viviendo. Un listado de servicios a dónde dirigir a la persona, si fuera el caso, para recibir apoyo, ayuda y sostén ante lo vivido y no resuelto o ante lo que está viviendo en el presente. Es decir, prestar toda ayuda posible que le permita sostener las posibles emociones y dificultades emergentes de la caja de pandora abierta al remembrar la historia vivida.

\section{Conclusiones}

La investigación de temas sensibles implica la utilización del método científico de una forma sistemática y rigurosa afrontando los desafíos que plantea el trabajo con seres humanos, sus sentimientos, experiencias y circunstancias. El trabajo con este tipo de temática supone comprender en profundi- 
dad y trabajar con delicadeza la dimensión personal y humana. Para llevar a cabo una investigación con temática sensible de una forma adecuada, pero sobre todo ética, es imprescindible comprender lo que el término sensible conlleva; esto supone añadir estrategias para afrontar las situaciones que pueden surgir durante el trabajo de campo en el momento del diseño de la investigación. En ese sentido, se debe contar con la dificultad de acceder a las vivencias de quienes participan y tener en cuenta la dimensión emocional de quien es investigado o investigada. También, tomar conciencia de aquello que la investigación implica para quien investiga; la puesta en práctica de estrategias que favorezcan la ventilación emocional y la desconexión con la investigación. Estos supuestos deben evitar la victimización secundaria y traumatización de quienes participan. También, la no - reproducción de las dinámicas violentas (por ejemplo, en la exigencia de deberes o el privilegio que ofrece tener información íntima sobre aquel que explica su historia) entre los investigadores y participantes. Las observaciones hasta ahora mencionadas, sin embargo, deben tornarse señales y oportunidades para el estudio; esto es, atender, comprender, explicar y actuar en consecuencia a lo que significa el hecho de que, por ejemplo, sea restringido el acceso a los participantes (nos cierren las puertas o no sepamos a dónde acudir para encontrar personas dispuestas a dar su testimonio), los silencios repetidos ante ciertas cuestiones cuando se está en el campo, las evasivas de los entrevistados a tratar temáticas concretas o el impacto emocional de la investigación para los que integran el trabajo. Para ello, es necesaria una revisión constante de las prácticas que se llevan a cabo, sea cual sea el método utilizado y en la disciplina en la cual se aplique (educación, psicología, sociología, etc.).

Esta investigación nos ha llevado a reflexionar en torno a cuestiones muy relevantes: la validez de las herramientas de las que disponemos para acceder al conocimiento, la sensibilidad de estas respecto al objeto de estudio y la ética de la investigación. Nuestro objetivo fue conocer la visión, percepción, pensamientos y sentimientos, es decir, el universo interior de los y las participantes respecto a un área de su vida a través de una entrevista en profundidad. Tras la realización de las entrevistas, transcripción y análisis de las mismas, pudimos comprobar que existe cierta información a la que no es posible acceder de forma directa. ¿Significa esto que nuestros instrumentos no son validos para alcanzar el conocimiento? $i \mathrm{O}$ podemos realmente con ellos representar la realidad de los y las participantes? Estas preguntas no deben ser ignoradas ni llevarnos a la frustración en la práctica investigadora; más bien, estos fenómenos deben ser descritos y posteriormente interpretados con mucha cautela, tratando de no realizar hipótesis injustas e injustificadas sobre los porqués de quienes participan (por ejemplo, al ocultar información), lo cual podría llevarnos a una subjetividad poco rigurosa. Es necesario un análisis en profundidad de este problema, así como del contexto social que lo acoge para ofrecer una respuesta exhaustiva y matizada de sus causas.

Teniendo en cuenta lo anteriormente mencionado, consideramos que la metodología cualitativa es muy beneficiosa cuando se trata de indagar en las experiencias privadas de las personas. Principalmente, porque nos permite introducir estrategias que favorecen la expresión emocional y el cuidado de la persona participante - convirtiéndose en un tipo de metodología sensible a aquello que se investiga -; captar sutilezas del discurso; tener en cuenta lo verbal y lo no verbal, lo que se dice y lo que se quiere silenciar. Nos ayuda a comprender, a través del relato y las reacciones, el impacto emocional de aquello que estamos investigando, más allá de los datos; nos permite poner voz, tono, expresión y rostro a las vivencias.

Por lo anteriormente mencionado, consideramos la importancia de abordar los fenómenos sociales desde el punto de vista de la ética de la investigación. Más aún cuando la investigación científica, sobre todo en el intento de respetar y proponer estrategias para alcanzar los fines constitutivos (justicia, bienestar y orden social) de la política social, tiene un impacto claro en la creación de políticas públicas (en igualdad, violencia de género, etc.) que atañen directamente a la vida de las personas.

Nos referimos a las políticas públicas, entendidas por Velásquez Gavilanes (2009) como

un proceso integrador de decisiones, acciones, inacciones, acuerdos e instrumentos, adelantado por autoridades públicas con la participación eventual de los particulares, y encaminado a solucionar o prevenir una situación definida como problemática. La política pública hace parte de un 
ambiente determinado del cual se nutre y al cual pretende modificar o mantener (p. 156).

No podemos, en tanto que hablamos de proceso, desatender que la misma tiene un carácter no solo cambiante; sino también intencional al marcarse sobre el qué y cómo actuar.

Asimismo, nuestras deliberaciones en cuanto a la ética de la investigación nos han llevado, en vez de a sólidas respuestas, a inciertas preguntas; ¿Cuáles son los límites éticos cuando tratamos con una temática sensible? ¿ Hasta qué punto podemos, de forma moral, adentrarnos e indagar en la intimidad de quien participa? ¿Deberíamos actuar si nuestra investigación implica la movilización de procesos emocionales o recuerdos traumáticos de quienes participan? ¿Qué relación guarda todo ello en las políticas sociales y públicas? El debate en torno a estas preguntas es una propuesta para futuras investigaciones en esta línea.
Recordando el último objetivo de nuestro trabajo - brindar un compendio de técnicas útiles en un trabajo con temática sensible - las consideraciones metodológicas que ofrecemos en este texto son estrategias que aspiramos a que puedan contribuir a ayudar a la comunidad académica a sortear o minimizar algunos de los obstáculos que pueden surgir al trabajar temáticas de gran sensibilidad (por ejemplo, acoso escolar, problemas emocionales, violencia de género, etc.). Por último, poner énfasis en la necesidad de permanecer constantes y con espíritu crítico, ser flexibles y adaptarnos a las circunstancias en nuestras investigaciones para evitar lo que Díaz Fernández et al. en 2012 afirman al exponer que los y las investigadores/as sociales no debemos dejar de explorar ambientes que puedan ser hostiles, pues esto nos haría caer en el riesgo de empobrecer nuestras disciplinas por la homogeneización de las rutinas éticas y de las metodologías más ortodoxas.

\section{Referências}

Alencar-Rodrigues, R., Cantera, L. M., \& Ojeda Ocampo Moré, C. (2014). Investigación sobre violencia de género en la pareja: Recomendaciones prácticas. Temas Em Psicologia, 22(1), 79-91. https://doi.org/10.9788/TP2014.1-07

Arón, A. M., \& Llanos, M. T. (2004). Cuidar los que cuidan: Desgaste profesional y cuidado de los equipos que trabajan con violencia. Sistemas Familiares, 1(2), 5-15.

Arranz, F. (2015). Meta-análisis de las investigaciones sobre violencia de género: El estado produciendo conocimiento. Athenea Digital, 15(1), 171-123. https://doi.org/10.5565/rev/athenea.1394

Bustelo, M., \& Lombardo, E. (2006). Los “marcos interpretativos" de las políticas de igualdad en Europa: Conciliación, violencia y desigualdad de género en la política. Revista Española de Ciencia Política, 40(14), 117-140.

Cantera, L. M. (2002). La relación asistencial como interacción social. In M. T. López Beltrán, M. J. Jiménez Tomé, \& E. M. Gil Benítez (EdS.), Violencia y gênero. Málaga: CEDMA.

Cantera, L. M. (2018). Violència domèstica i delinqüència contra les dones. Barcelona: Universitat Autònoma de Barcelona.

Castellano, A. M. (1998). Problematic defínition of the qualitative methodologies. Espacio Abierto, 7(3), 443-459.

Castillejo, A. (2005). Las texturas del silencio:Violencia, memoria y los límites del quehacer antropologico. Empiria, (9), 39-59. https://doi.org/10.5944/empiria.9.2005.1003

Catela, L. (2000). De eso no se habla: Cuestiones metodológicas sobre los límites del silencio en entrevistas a familiares de desaparecidos políticos. Historia, Antropología y Fuentes Orales, 2(24), 69-75.

Catela, L. (2004). Conocer el silencio: Entrevistas y estrategias de conocimiento en situaciones límites. Oficios Terrestres, (15-16), 42-54.

Declan, F. (2014). Doing sensitive research sensitively: Ethical and methodological issues in researching workplace bullying. International Journal ofQualitative Methods, 13(1), 19-36. https:// doi.org/10.1177/160940691401300108

Díaz Facio-Lince, V. E., Molina Jaramillo, A. N., \& Marin, M. A. (2014). Significados, silencios y olvidos asociados a la experiencia del desplazamiento forzado. Revista de Psicología Universidad de Antioquia, 6(2), pp. 9-26.

Díaz Fernández, A., Ruiz Rodríguez, L., González Agudelo, G., \& Gallardo García, R. (2012). Guía para investigar temas sensibles. Cádiz. Recuperado de http://www.uca.es/recursos/doc/Unidades/Unidad_Innovacion/Actuaciones/ANEXOS_2011_2012/1547550019_2211201213936.pdf 
Goodrum, S., \& Keys, J. L. (2007). Reflections on two studies of emotionally sensitive topics: Bereavement from murder and abortion. International Journal of Social Research Methodology, 10(4), 249-258. https://doi. $\operatorname{org} / 10.1080 / 13645570701400976$

Lee, R. (1993). Doing research on sensitive topics. London: Sage.

López Estrada, R. E., \& Deslauriers, J.-P. (2011). La entrevista cualitativa como técnica para la investigación en trabajo social. Margen, (61), 1-19.

McCosker, H. (1995). Women's conceptions of domestic violence during the childbearing years (Masters thesis). Brisbane:, Queensland University of Technology.

McCosker, H., Barnard, A., \& Gerber, R. (2001). Undertaking sensitive research: issues and strategies for meeting the safety needs of all participants. Forum: Qualitative Social Research, 2(1), 22. https://doi.org/10.17169/FQS$-2.1 .983 \mathrm{de}$

Organizacion Mundial de la Salud - OMS. (1999). Dando prioridad a las mujeres: Recomendaciones éticas y de seguridad para la investigación sobre violencia doméstica contra las mujeres. Geneva: Organizacion Mundial de La Salud.

Pita, S., \& Pértegas, S. (2002). Investigación cuantitativa y cualitativa. Cadena Atención Primaria, 9, 76-78.

Quiceno, N. (2008). Puesta en escena, silencios y momentos del testimonio: El trabajo de campo en contextos de violencia. Estudios Políticos, (33), 181-208.

Quiñones, P., Cantera, L., \& Ojeda, C. (2013). La violencia relacional en contextos laborales que trabajan contra la violência. In L. Cantera, S. Pallarès \& C. Selva (EdS.), Del malestar al bienestar laboral (pp. 77-105). Barcelona: Amentia.

Ramírez-Pereira, M. (2017). Metodologías cualitativas de lo sensible en contextos de salud pública. Enfermería: Cuidados Humanizados, 6(num espec.), 25-30. https://dx.doi.org/10.22235/ech.v6iespecial.1447

Romero, J. P. A. (2008). El investigador ante lo indecible y lo inenarrable. Nómadas, 29, 20-33.

Sandín, P. (2003). Tradiciones en la investigación cualitativa. In M. Paz (Ed.), Investigación cualitativa en educación fundamentos y tradiciones, México, DF: MacGraw Hill.

Schraiber, L., Oliveira, A., \& Couto, T. (2009). Violência e saúde: Contribuições teóricas, metodológicas e éticas de estudos da violência contra a mulher. Cadernos de Saúde Pública, 25(suppl 2), s205-s216. https://doi.org/10.1590/ S0102-311X2009001400003

Sieber, J., \& Stanley, B. (1988). Ethical and professional dimensions of socially sensitive research. American Psychologist, 43(1), 49-55.

Varguillas, C., \& Ribot de Flores, S. (2007). Implicaciones conceptuales y metodológicas en la aplicación de la entrevista en profundidad. Laurus, 13(23), 249-262.

Velásquez Gavilanes, R. (2009). Hacia una nueva definición del concepto “política pública” . Desafios, (20), 149-187. https://revistas.urosario.edu.co/index.php/desafios/article/view/433/377

\section{Alicia Pérez-Tarres}

Universitat Autònoma de Barcelona, Facultad de Psicología, Departamento de Psicología Social, Barcelona. Espanha.

E-mail: ptt.alicia@gmail.com

https://orcid.org/0000-0002-3686-1430

\section{Leonor María Cantera Espinosa}

Universitat Autònoma de Barcelona, Facultad de Psicología, Departamento de Psicología Social, Barcelona. Espanha.

E-mail: leonor.cantera@uab.es

https://orcid.org/0000-0002-4541-5993 


\section{Karine David Andrade Santos}

Universidade Federal de Sergipe, Facultad de Psicología, São Cristóvão - SE. Brasil.

E-mail: psimulti@gmail.com

(iD) https://orcid.org/0000-0001-9951-9539

Joilson Pereira da Silva

Universidade Federal de Sergipe, Facultad de Psicología, São Cristóvão - SE. Brasil.

E-mail: joilsonp@hotmail.com

(iD) https://orcid.org/0000-0001-9149-3020

Endereço para envio de correspondência:

Universitat Autònoma de Barcelona, Facultad de Psicología Social. Edifici B. Despatx B5/040 Campus de La UAB. 08193. Bellaterra (Cerdanyola Del Vallès). Barcelona, España. 34-93-581-2189.

Recebido:28/06/2019

Aceito: 05/08/2019

Received:06/28/2019

Approved: 08/05/2019

Recibido:28/06/2019

Aceptado: 05/08/2019

Como citar: Pérez-Tarres, A., Cantera Espinosa, L. M., Santos, K. D. A., \& Silva, J. P. (2019). Considerações metodológicas sobre investigação sensível em metodologia qualitativa. Psicologia: Ciência e Profissão, 39(n.spe 2), 112-124. https://doi.org/10.1590/1982-3703003225746

How to cite: Pérez-Tarres, A., Cantera Espinosa, L. M., Santos, K. D. A., \& Silva, J. P. (2019). Consideraciones metodológicas sobre investigaciones sensibles en metodología cualitativa. Psicologia: Ciência e Profissão, 39(n.spe 2), 112-124. https:// doi.org/10.1590/1982-3703003225746

Cómo citar: Pérez-Tarres, A., Cantera Espinosa, L. M., Santos, K. D. A., \& Silva, J. P. (2019). Methodological considerations for sensitive research on qualitative methodology. Psicologia:Ciênciae Profissão, 39(n.spe 2), 112-124. https://doi.org/10.1590/1982-3703003225746 